\title{
How Symbolic Experience Shapes Children's Symbolic Flexibility
}

\author{
Emily E. Thom \\ California State University, Northridge
}

\author{
Catherine M. Sandhofer \\ University of California, Los Angeles
}

\begin{abstract}
The current experiments asked whether children with dual-symbolic experience (e.g., unimodal bilingual and bimodal) develop a preference for words like monolingual children (Namy \& Waxman, 1998). In Experiment 1, ninety-five 18- and 24-month-olds, with monolingual, unimodal bilingual, or bimodal symbolic experience, were tested in their willingness to treat digitized sounds as referents. In Experiment 2, forty-seven 24month-olds, with the same types of symbolic experience, were tested in their willingness to treat novel words as referents. Monolingual children performed in ways indicative of a growing preference for words, whereas children with dual-symbolic experience performed in ways indicative of consistency in symbolic flexibility over time. Results suggest that the developmental trajectory of children's symbolic flexibility might depend on their symbolic experience.
\end{abstract}

Monolingual children's symbolic flexibility varies across early development. Between birth and 2 years of age, young monolingual children represent the world, and express themselves, in a myriad of ways. Between 12 and 18 months of age, they treat multiple symbolic forms (e.g., words, nonverbal sounds, hand signs, pictograms) as labels for referents (Hollich et al., 2000; Namy, 2001; Namy \& Waxman, 1998; Woodward \& Hoyne, 1999). They become more conservative in their mapping behavior over time, however. By 20-26 months of age, they develop a preference for words over other symbolic forms, and treat only symbols of that form as labels for referents (Namy \& Waxman, 1998; Woodward \& Hoyne, 1999). However, this preference for words is only temporary: Monolingual children's symbolic flexibility returns by the time they are 4 years of age and remains present thereafter (Namy, Campbell, \& Tomasello, 2004).

In this study, we sought to better understand the factors contributing to children's symbolic flexibility (or lack thereof). We explored the possibility that, at any point in early development, the extent of children's symbolic flexibility depends on the breadth of the cumulative symbolic experience (i.e.,

The authors would like to thank Elizabeth Abate, Melissa Arce, Whitney Brammer, Natalie Carlos, Aimee Dodge, Megan Galligan, Ashley Evans, Angela Lopez, Jessica McNally, Jennifer Wong, and Sabrina Zakutinsky for their help in completing this project.

Correspondence concerning this article should be addressed to Emily E. Thom, Department of Child and Adolescent Development, California State University Northridge, 280E Sequoia Hall, 18111 Nordhoff Street, Northridge, CA 91330. Electronic mail may be sent to emily.thom@csun.edu. the type or number of symbolic forms to which they have been regularly exposed). We asked whether different types of symbolic experience (e.g., monolingual, unimodal bilingual, bimodal) might lead to differences in the developmental trajectory of children's symbolic flexibility.

\section{Symbolic Flexibility Varies Across Early Development}

Early on, children exhibit remarkable symbolic flexibility. For example, 12-month-olds map nonword verbal sounds (e.g., a tongue click) and digitized sounds (e.g., a beep) when they are provided additional cues to reference (Hollich et al., 2000). Similarly, 17-month-olds map novel words (e.g., toma), hand signs (e.g., a dropping motion), nonverbal sounds (e.g., a two-toned beep), and pictograms (e.g., a colorful block image) to referents when the symbols are embedded in common linguistic frames (Namy, 2001).

By 2 years of age, monolingual children's symbolic flexibility is superseded by a strong preference for words as a symbolic form (Namy \& Waxman, 1998; Namy et al., 2004). Woodward and Hoyne (1999) elegantly demonstrated this phenomenon with their comparison of 13- and 20-month-olds' willingness to treat novel words or sounds as labels for novel objects. The experimenters labeled the objects for children by embedding the symbols within common linguistic frames (e.g., "Look it's a

(C) 2013 The Authors

Child Development (C) 2013 Society for Research in Child Development, Inc. All rights reserved. 0009-3920/2013/xxxx-xxxx

DOI: $10.1111 /$ cdev. 12147 
toma. See? That's the toma." or "Look at this. [Squeak]. Yeah, see it? [Squeak]."), and then tested proper mapping of the labels using a multiplechoice procedure (e.g., "Can you get the toma?" or "Can you get one of these? [Squeak]."). Results indicated that children's mapping behavior depended on their developmental level: Whereas 13-month-olds successfully mapped both novel words and sounds to novel objects, 20-month-olds mapped only words to novel objects.

Namy and Waxman (1998) found a similar pattern of results with their comparison of 18- and 26-month-olds' willingness to treat novel words or novel hand signs as labels for categories of familiar objects. Whereas 18-month-olds successfully mapped both words and hand signs to object categories, 26-month-olds mapped only words to object categories. Interestingly, however, the authors found in a second experiment that 26-month-olds could successfully map hand signs to object categories, but only when given extended training in the use of hand signs as labels for referents. In a follow-up study, Namy et al. (2004) found that 4-year-olds could successfully map hand signs to categories of familiar objects without extended training. Thus, it appears that monolingual children's preference for words peaks around their second birthdays, and subsides sometime thereafter.

\section{Symbolic Experience Shapes Symbolic Flexibility}

Why does symbolic flexibility of monolingual children vary across early development? Namy et al. (2004) have argued that a temporary preference for words serves an important function as part of the process of verbal language acquisition: Rigidly differentiating words from other potential symbolic forms allows monolingual children to move beyond the initial phase of using symbols to represent ideas in a one-to-one fashion, and thus to make inroads into the complexities of the to-belearned linguistic system. According to the authors, monolingual children's preference for words subsides as they make sufficient progress in verbal language acquisition and gain sufficient practice using nonword symbolic forms. As this occurs, a preference for words becomes less necessary to promote their verbal language acquisition and less relevant to their symbolic experience.

Consistent with this account is the fact that children reach a number of important milestones of verbal language acquisition around 2 years of age, when their preference for words is peaking. For example, at approximately 18 months of age, many children enter into a period of rapid vocabulary growth (Heibeck \& Markman, 1987; Mervis \& Bertrand, 1995) and begin transitioning from oneword to two-word speech (Bloom, 1973; Nelson, 1973). Furthermore, by 4 years of age, when their symbolic flexibility has returned to previous levels, monolingual children have amassed sizable productive vocabularies (of approximately 1,000 words; Fenson et al., 1991), and have begun using nonword symbolic forms (e.g., maps, pictures, models, and written language) to represent the world and to express themselves (Piaget, 1923/1997).

In this study, we propose a new account of monolingual children's temporary preference for words. We posit that their symbolic flexibility depends on their cumulative symbolic experience throughout early development, not just at a particular moment in time. In doing so, we reframe their preference for words as being highly adaptive to their symbolic experience, rather than simply, coincidentally, functional for their verbal language acquisition. Most children are inundated by spoken words from birth. By some estimates, they hear between 300 and 500 words an hour (Behrens, 2006). For monolingual children, most of these words are from a single, spoken language. As the result of this experience, their representational systems may "tune" to words over time, just as their other systems attune to the stimulus that has historically mattered for learning (Eimas, Sigueland, Jusczyk, \& Vigorito, 1971; Palmer, Fais, Golinkoff, \& Werker, 2012; Smith, Jones, Landau, GershkoffStowe, \& Samuelson, 2002; Tees \& Werker, 1984). However, as monolingual children's symbolic experience continues to increase, and diversify, their representational systems may adapt (and readapt) accordingly, leading to a reemergence of symbolic flexibility over time.

The proposed account implies that a temporary preference for words is only one of a number of possible developmental outcomes. Based on our argument, differences in the breadth of symbolic experience should lead to differences in the developmental trajectory of symbolic flexibility. If "narrow" symbolic experience, such as that of monolingual children, leads to a preference for a single symbolic form, then "broader" symbolic experience, such as that of bilingual children, should maintain some level of symbolic flexibility throughout early development. Thus, if the account is correct, we should expect bilingual children to exhibit greater symbolic flexibility than monolingual children in the later stages of early development (i.e., between approximately 2 and 3 years of age). 


\section{Bilingual Symbolic Experience Leads to Different Developmental Trajectories}

Indeed, some studies have provided preliminary support for our hypothesis. For example, Petitto et al. (2012) compared the neural activity of 4- and 12-month-old monolingual and bilingual infants as they processed phonetic stimuli from their native language as well as from a non-native language. The authors found differences in the 12-month-olds' neural activity according to their symbolic experience: Whereas the older monolingual infants showed distinct patterns of activity when processing native phonetic stimuli versus non-native phonetic stimuli, the older bilingual infants showed similar patterns of activity when processing both native and non-native phonetic stimuli. These findings are significant as they suggest that the unimodal bilingual representational systems do not attenuate in the same manner as the monolingual representational systems (cf. Eimas et al., 1971; Tees \& Werker, 1984). To explain their findings, Petitto et al. authors offered the perceptual wedge hypothesis:

The number of input languages to which a young baby is exposed can serve as a kind of "perceptual wedge." Like a physical wedge that holds open a pair of powerfully closing doors, exposure to more than one language holds open the closing "doors" of the human baby's typical developmental perceptual attenuation processes, keeping language sensitivity open for longer (witness the older bilingual babies' more "open" sensitivity to non-native phonetic contrasts as compared to monolingual babies). (p. 11).

Put simply, the authors argue that unimodal bilingual experience disrupts the brain's typical attenuation processes, making the bilingual infants' brain more expansive and resilient, and more likely to process native and non-native phonetic stimuli in the same way.

Additional support for our hypothesis comes from a comparison of monolingual and bilingual children's graphical representation abilities. AdiJapha, Berberich-Artzi, and Libnawi (2010) compared the ability of 7-year-old monolingual and unimodal bilingual children to draw impossible objects, which were hybrids of two known objects (e.g., a "giraffe-flower"). The authors found that there were more signs of interrepresentational flexibility (i.e., the ability to entertain multiple representations at the same time) in the drawings of unimodal bilingual children than in the drawings of monolingual children. For example, the drawings of unimodal bilingual children frequently depicted an imagined hybrid of the two known objects, whereas the drawings of monolingual children frequently depicted the known objects separately.

\section{Types of Bilingual Symbolic Experience}

The findings of Petitto et al. (2012) and AdiJapha et al. (2010) suggest that differences in the breadth of symbolic experience might lead to differences in the developmental trajectories of children's representational, perceptual, and cognitive systems. However, their findings are limited to children with only one type of bilingual symbolic experience. The unimodal bilingual children in Petitto et al. and Adi-Japha et al. were trained in two symbolic forms within the same modality (e.g., spoken English and spoken Spanish). It is yet unclear how the authors' findings might apply to bimodal bilingual children, trained in two symbolic forms in different modalities (e.g., spoken English and American sign language [ASL]), as there are key differences between these types of dual-symbolic experience (Namy, Acredolo, \& Goodwyn, 2000; Pyers, Gollan, \& Emmorey, 2009). Nevertheless, there are reasons to think that both types of bilingual symbolic experience might lead to symbolic flexibility in the later stages of early development-albeit in different ways.

\section{Unimodal Bilingualism}

Individuals with unimodal bilingual symbolic experience represent and control the use of two similar symbolic forms that involve the same perceptual and motor systems. These systems may have some phonological and lexical overlap. Because unimodal bilinguals are physically unable to use both symbolic forms at the same time, they must code switch, that is, transition between the symbolic forms sequentially. To do this, they must perform the difficult task of actively selecting the desired symbolic form as well as suppressing the undesired symbolic form. It is thought that code switching "exercises" the general cognitive systems, making unimodal bilinguals-young and oldmore efficient than monolinguals at monitoring their environments, controlling their attention, and using attentional cues (Bialystok \& Shapero, 2005; Comeau \& Genesee, 2001; Costa, Hernández, \& Sebastián-Gallés, 2008).

Symbol learning contexts are rich with information that can serve as cues to a to-be-learned 
symbol and its intended referent. Such cues include the speaker's use of common linguistic frames, eye gaze, gesturing, and movement of an object (Baldwin, 1993; Samuelson \& Smith, 2005; Scaife \& Bruner, 1975; Tomasello, 1992). Some previous research (e.g., Yow \& Markman, 2011) has suggested that as the result of their enhanced cognitive abilities, unimodal bilingual children are better able to use these cues to solve the problem of reference in wordlearning contexts. It is possible that this advantage extends to contexts in which they are asked to learn symbols from untrained forms as labels for referents, making them more likely to map the symbols to their proper referents, and thus, more likely to exhibit intact symbolic flexibility in the later stages of early development.

\section{Bimodal Bilingualism}

Individuals with bimodal bilingual symbolic experience represent and control two symbolic forms that involve different perceptual and motor systems. These systems have no phonological or lexical overlap. Thus, in contrast to unimodal bilinguals, bimodal bilinguals can code blend, that is, use both symbolic forms simultaneously (Emmorey, Borinstein, Thompson, \& Gollan, 2008; Emmorey, Luk, Pyers, \& Bialystok, 2008). Although bimodal bilinguals experience some difficulty in selecting the desired symbolic form and suppressing the undesired symbolic form, code blending does not appear to exercise the general cognitive systems in the same way code switching does (Kovelman et al., 2009; Pyers et al., 2009). Bimodal bilinguals show less of an advantage (or none at all) over monolinguals in monitoring their environments, controlling their attention, or using attentional cues (Emmorey, Borinstein, et al., 2008; Emmorey, Luk, et al., 2008). For these reasons, if bimodal bilingual symbolic experience leads to intact symbolic flexibility in the later stages of early development, it is likely to do so in unique ways.

One such way might be through promoting children's awareness of the referential and communicative function of all symbolic forms, not just those to which they have been regularly exposed. Research has shown that comparing diverse instances of a category leads to increased conceptual abstraction and higher level generalizations (e.g., Namy \& Gentner, 2002; Oakes \& Ribar, 2005; Waxman, 2003; Waxman \& Klibanoff, 2000). In contexts where they are asked to treat symbols from an untrained form as labels for referents, children with bimodal bilingual symbolic experience might benefit from their exposure to and use of diverse symbolic forms at the same time. They might perceive the symbols not as foreign, but as new instances of a conceptually abstract category (e.g., "modes of symbolic reference"), and thus be more likely to generalize their previous symbol learning experience to new symbol learning contexts.

Bimodal bilingual children's tendency to make higher level generalizations in new symbol learning contexts might also be increased as the result of their exposure to certain key properties of multiple symbolic forms. Colunga and colleagues (Brojde \& Colunga, 2006; Colunga \& Smith, 2002) have argued that children will map any symbol to a referent as long as that form has historically possessed the properties of cue validity and systematicity. A symbolic form has high cue validity if it has been frequently and consistently associated with a particular object category (i.e., a one-to-one mapping of a specific word or gesture to an object category). A symbolic form is systematic if many signals co-occur with the symbols themselves (e.g., words are systematic because other signals, such as speaking words from the mouth or pointing to the object, are "bundled" with them). According to the authors, learning individual symbols exposes children to the cue validity and systematicity of the entire symbolic form to which they belong. In turn, these features help children to generalize their previous symbol learning experience to new symbol learning contexts. In the case of monolingual children, this generalization occurs within their trained symbolic form. For bimodal bilingual children, this generalization might occur across symbolic forms.

\section{Current Experiments and Hypotheses}

Thus, there are a number of reasons to believe that bilingual symbolic experience might lead to intact symbolic flexibility in the later stages of early development. We tested this possibility in a series of two experiments.

\section{Experiment 1}

In Experiment 1, we compared the symbolic flexibility of younger and older children (18 and 24 months of age, respectively), with monolingual, unimodal bilingual, and bimodal symbolic experience. In a sound label task, we labeled novel objects for children using digitized sounds (e.g., a click), and then tested children's proper mapping of the labels using a multiple-choice procedure. 
Because any differences in symbolic flexibility should emerge only in the later stages of early development (Namy \& Waxman, 1998; Woodward \& Hoyne, 1999), we were particularly interested in the performance of older children in Experiment 1. We predicted that older monolingual children would perform on the sound label task in ways indicative of a preference for words as a symbolic form-in other words, they would fail to map the sound labels to the novel objects. Our predictions were less specific for older children with dualsymbolic experience. For reasons already outlined, we predicted that at least one group of these children would perform in ways indicative of having intact symbolic flexibility-in other words, they would successfully map the sound labels to the novel objects. However, we were aware of three possible patterns of results that fit within this criterion, and each implied different explanations of such mapping behavior.

If, on the sound label task, older children with unimodal bilingual experience mapped the sound labels to the novel objects, but older children with bimodal bilingual experience did not, the implication would be that their symbolic flexibility had resulted from abilities uniquely associated with unimodal bilingual experience (e.g., greater efficiency in environmental monitoring, attentional control, use of social and contextual cues to reference). Conversely, if older children with bimodal symbolic experience mapped the sound labels to the novel objects, but older children with unimodal bilingual experience did not, the implication would be that their symbolic flexibility had resulted from abilities uniquely associated with bimodal bilingual experience (e.g., heightened awareness of the function of all symbols; increased tendency to make conceptual abstractions, and higher level generalizations).

Finally, if both older children with unimodal bilingual symbolic experience and older children with bimodal symbolic experience mapped the sound labels to the novel objects, the implication would be that their symbolic flexibility had resulted from either (a) abilities uniquely associated with each type of dual-symbolic experience or (b) abilities associated with both types of dual-symbolic experience. If the former explanation were accurate, then it would be the case that different abilities had led to the same liberal mapping behavior. If the latter explanation were accurate, then it would present the possibility that there are more similarities between the experience and abilities of unimodal bilinguals and bimodal bilinguals than previously thought.

\section{Experiment 2}

Experiment 2 was conducted as a control condition for Experiment 1. If, as predicted, we observed differences in performance on the sound label task between the symbolic experience groups, it would be possible that they had resulted not from differences in symbolic flexibility, but from differences in their willingness or ability to treat symbols from any form (trained or untrained) as labels for referents - a general symbolic ability per se. To rule this out, we compared the general symbolic ability of older children (24 months of age) with monolingual, unimodal bilingual, and bimodal symbolic experience. In a word label task, we labeled novel objects for children using novel, English-like words (e.g., dax), and tested children's proper mapping of the labels using the same multiple-choice procedure used in Experiment 1.

Although indeed a possibility, we had no reason to believe that differences in symbolic experience might also lead to differences in general symbolic ability based on our account; thus, we predicted that all children in Experiment 2-each of whom had been regularly exposed to English words as a symbolic form - would easily map the novel words to the sound labels and perform equally well on the word label task.

\section{Dual-Symbolic Experience Groups}

In Experiments 1 and 2, we included two groups of children with dual-symbolic experience: (a) those with unimodal bilingual experience, who had been regularly exposed to spoken English and spoken Spanish, and (b) those with bimodal symbolic experience, who had been regularly exposed to spoken English and some form of a signed language (e.g., ASL). It is important to note that children in the bimodal symbolic experience group were not true bimodal bilinguals, as their primary language was spoken English, and some had not been exposed to the grammatical elements of the signed language. We were aware that for this reason, the results of our study would represent an important, but preliminary, step toward understanding how different types of bilingual symbolic experience might impact the developmental trajectories of children's symbolic flexibility and their representational systems. 


\section{Experiment 1}

\author{
Method
}

\section{Participants}

Participants were recruited through a shared laboratory database as well as through school facilities in the greater Los Angeles area. Younger and older children were placed into the monolingual, unimodal bilingual, and bimodal symbolic experience groups based on their parents' responses on a specially designed questionnaire.

Parental questionnaire and inclusion criteria. The parental questionnaire consisted of three parts. The first part asked parents to list the languages to which their child was regularly exposed and to estimate the percentage of time their child was exposed to each language on a daily basis. The second part asked parents to indicate if their child had been exposed, or was currently being exposed, to a signed language on a regular basis (e.g., ASL or baby sign). "Baby sign" refers to variations in a teaching technique wherein young hearing children are exposed to hand signs to facilitate communication before their verbal expression takes off. There are many variations in baby sign; common to all is an emphasis on the use of signs with spoken word, as well as on the semantic aspect of the symbolic form (although ASL has formal grammatical elements, they are not commonly trained as part of the baby sign technique). If parents' answer to this question was "yes," they were asked to indicate the age of their child when this exposure had begun and the age of their child when this exposure had ended, if applicable. Parents were also asked to indicate who had exposed their child to a signed language. The third part of the questionnaire asked parents to estimate their child's sign vocabulary size by checking the signs their child produced on a list of 84 common nouns and verbs (e.g., book, banana).

Children were considered monolingual if, based on parental report, they (a) were being exposed to English as a primary language in their homes or school facilities (if enrolled) and (b) were not currently being exposed to a signed language on a regular basis. Children were considered unimodal bilingual if they (a) were being exposed to English and Spanish in their homes or school facilities at approximately equal frequencies and (b) were not currently being exposed to a signed language on a regular basis. Children were considered to have bimodal symbolic experience if they (a) were being exposed to English as a primary language in their homes and school facilities and (b) were currently being exposed to a signed language.

Additional measures. English and Spanish versions of the MacArthur-Bates Communicative Development Inventory: Words and Sentences (MCDI; Fenson et al., 1993) were used to estimate children's productive vocabularies.

Monolingual children. Table 1 presents a thorough summary of participant characteristics in each of the symbolic experience groups from Experiment 1. As can be seen, 31 monolingual children were included in Experiment 1. Sixteen of these children were younger $(M=18.14$ months), and 15 were older $(M=24.5$ months). Although English was the primary language for all monolingual children $(M=92.19 \%$ exposure), some had very limited exposure to other languages. Furthermore, many had small sign vocabularies despite having no regular exposure to a signed language (for a discussion of spontaneous signing among monolingual children, see Acredolo \& Goodwyn, 1988; Iverson, Capirci, Longobardi, \& Caselli, 1999).

Unimodal bilingual children. Twenty-nine unimodal bilingual children were included in Experiment 1. Fourteen of these children were younger $(M=17.06$ months), and 15 were older $(M=$ 25.14 months). Although English and Spanish were the primary languages for all unimodal bilingual children $(M=54.79 \%$ English exposure, $M=44.58 \%$ Spanish exposure), some had very limited exposure to other languages. Like their monolingual counterparts, it was fairly common for unimodal bilingual children to have small sign vocabularies.

Sources of language exposure. For the unimodal bilingual symbolic experience group, the sources of English and Spanish exposure varied from child to child. The majority of unimodal bilingual children were exposed to both symbolic forms in their homes and school facilities. Of these children, most were exposed to both symbolic forms by all caregivers (e.g., mother, father, and teachers spoke both English and Spanish with them). Some children were exposed to one symbolic form by one caregiver and the other symbolic form by another caregiver (e.g., mother spoke English with them and father spoke Spanish with them). Other children were exposed to one symbolic form in their homes and the other symbolic form in their school facilities (e.g., mother and father spoke Spanish with them at home, and teachers spoke English with them at school).

Children with bimodal symbolic experience. Thirtyfive children with bimodal symbolic experience were included in Experiment 1. Twenty-one of these children were younger $(M=17.25$ months), 


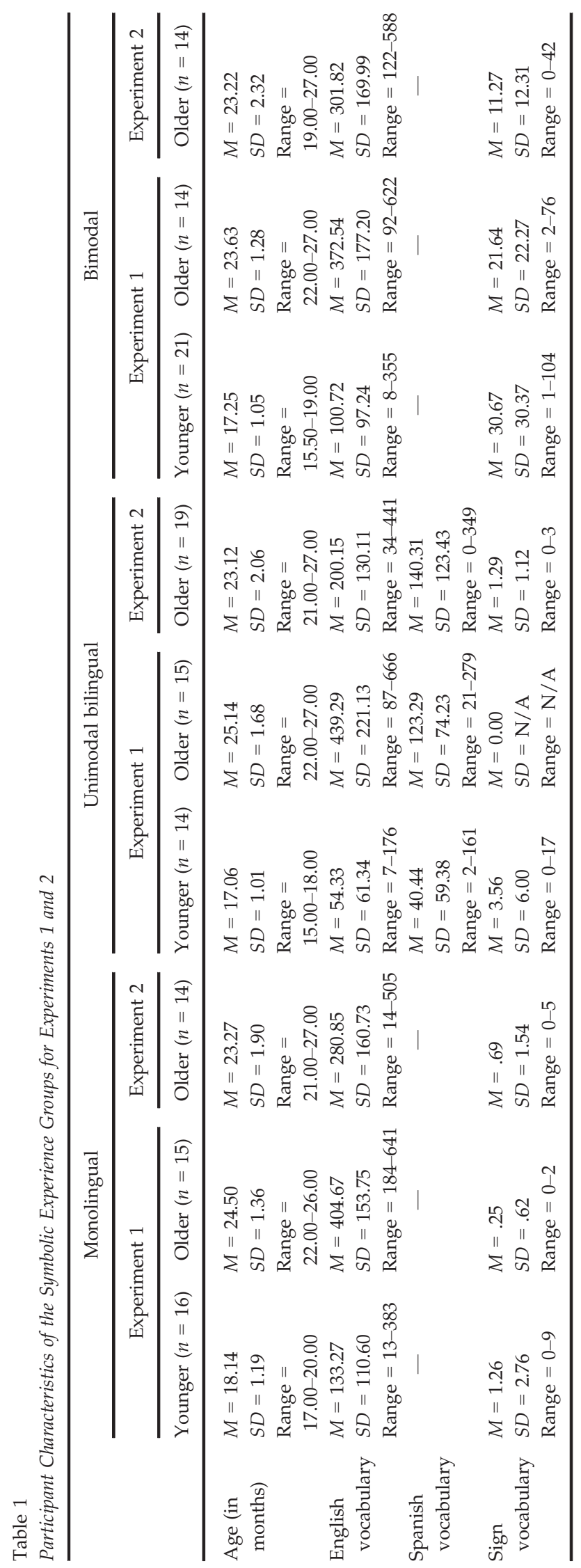

and 14 were older $(M=23.63$ months). Recall that children in the bimodal symbolic experience groups in this study were not true bimodal bilinguals. Thus, despite regular exposure to a signed language, their primary language was still English ( $M=93.92 \%$ exposure). Some children also had very limited exposure to other languages. As expected, children in this group had sizable sign vocabularies in comparison to children in the other symbolic experience groups.

Sources of language exposure. For the bimodal symbolic experience group, the sources of English and signed language exposure differed from child to child. The majority of children with bimodal symbolic experience were exposed to both symbolic forms in their homes and school facilities, if enrolled (e.g., mother, father, and teachers spoke English and used signs with them). Of these children, most had taken part in "Mommy and $\mathrm{Me}^{\text {"- }}$ type classes that trained signs for use in everyday life. Some had been trained in baby sign through books or videos. Other children were exposed to both symbolic forms at home, but only one symbolic form at school (e.g., mother and father spoke English and used signs with them, and teachers spoke English with them). A few children were exposed to one symbolic form at home, and both symbolic forms at school (e.g., mother and father spoke English with them, teachers spoke English and used signs with them). The signed language to which they had been exposed also differed from child to child. Some had been exposed to unmodified ASL, some had been exposed to modified ASL, and some had been exposed to idiosyncratic gestures created by their parents or teachers.

\section{Materials}

Familiar and novel objects. Twelve familiar objects and 16 novel objects were used in the pretest and sound label task. The familiar objects were chosen because they represented words that children typically know at 16-24 months of age (Fenson et al., 1993). The pool of familiar objects included: keys, a plate, a toy bottle, a small hat, a ball, a plastic plate, a rubber ducky, a book, a child's shoe, a bracelet, a toy cat, and a toy dog. The novel objects were created to look like unfamiliar toys to pique and maintain children's interest in them. They were three-dimensional and approximately 9 cubic in. in size. They were constructed from a variety of materials (e.g., wood, clay, plastic, and felt) and painted in bright colors. Figure 1 depicts examples of the 
Training (x 2):

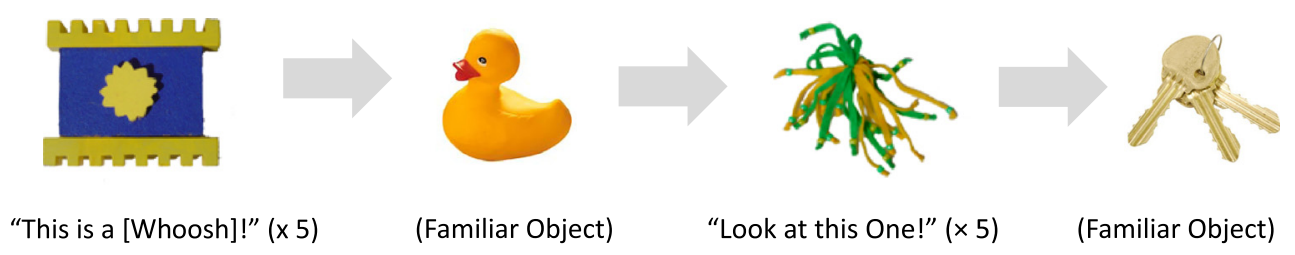

Testing:

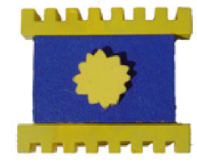

(Labeled Novel Object)



(Unlabeled Novel Object)

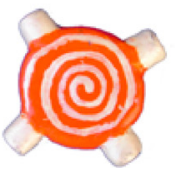

(Distracter Novel Object)

"Can you give me the [Whoosh]?"

Figure 1. Training-testing trial procedure for the sound label task in Experiment 1.

familiar and novel objects used in the pretest and sound label task.

Sounds. Four digitized sounds were used as labels for the novel objects in the sound label task. The sounds were chosen to be unfamiliar to the children. They could be roughly described as a "whoosh," "bang," "whistle," and "click." They were played using Microsoft PowerPoint software on a laptop computer, which was hidden from the children's view during the training and testing trials.

\section{Procedure}

The pretest and sound label task were conducted with individual children in a laboratory playroom or a quiet corner of their school facility. Often, the pretest and sound label task took place at a childsize table, with children seated opposite to the experimenter. If caregivers were present, they were seated next to their children. At times, the children sat in their parents' laps. Caregivers were asked to encourage their child to participate but refrain from labeling any of the objects or helping their child answer any questions during testing trials.

Pretest. The pretest was designed to ensure that children were capable of meeting the demands of the sound label task as well as to familiarize the children with its procedure. The pretest used three to six familiar objects, randomly selected for each child from the pool of 12 . The pretest and sound label task were always conducted in English.

The experimenter began the pretest by placing a single familiar object in the middle of the table. Children were allowed to handle the object briefly (approximately 5-10 s). The experimenter then pointed to the object and labeled it by saying, "This is a cat." or "Look! A cat." The experimenter repeated the label five times in total, using varied linguistic frames. After labeling, the experimenter removed the labeled familiar object from the table. Next, the experimenter placed a second familiar object in the middle of the table. Again, children were allowed to handle the object briefly. The experimenter then pointed to the object and directed children's attention to it by saying, "Look at this one!" or "Oh! This one." This was repeated five times in total. The familiar object was never labeled. The unlabeled familiar object was then removed from the table.

The experimenter then placed three objects in the middle of the table: (a) the labeled familiar object, (b) the unlabeled familiar object, and (c) a distractor familiar object (a previously unseen familiar object). Children were allowed to handle the objects briefly. The experimenter then asked children to select the labeled familiar object from the array by saying, "Can you give me the cat?" or "Which one of these 
is the cat?" Only neutral feedback (e.g., "Thank you!") was provided to children when they made their selection.

If children correctly selected the labeled familiar object, they moved on to the sound label task. However, if they incorrectly selected the unlabeled or distractor familiar object, the pretest procedure was repeated for a second time, using new familiar objects. If children continued to select incorrectly, they were deemed incapable of meeting the demands of the sound label task, and thus excluded from participation in the experiment.

Excluded participants. Eleven children were excluded from Experiment 1 for failing the pretest. An additional 19 children were excluded due to fussiness during the sound label task (described below). Of these 19, 9 would have been included in the monolingual symbolic experience group, 3 would have been included in the unimodal bilingual symbolic experience, and 7 would have been included in the bimodal symbolic experience group. These children were not included in the calculation of final sample sizes, descriptive analyses of participant characteristics, or inferential analyses of performance on the sound label task.

Sound label task. The sound label task consisted of four training-testing trials. Three novel objects and two familiar objects were randomly selected, without replacement, for each trial from the pools of 16 and 12 objects, respectively. Furthermore, the sounds used to label the novel objects were randomly selected, without replacement, for each trial from the pool of four sounds. This resulted in each child experiencing four unique sound-novel object pairings across the four training-testing trials.

Figure 1 depicts a single training-testing trial. As can be seen, the experimenter began each trainingtesting trial by placing a single novel object in the middle of the table. As in the pretest, children were allowed to handle the object briefly. The experimenter then pointed to the novel object and labeled it with a sound. The sound labels were played from the hidden laptop computer, and were always embedded within speech. For example, the experimenter said, "This is the [whoosh]." or "This is called a [whoosh]." The novel object was labeled five times in total, using varied linguistic frames. The labeled novel object was then removed from the table.

Campbell and Namy (2003) demonstrated that regardless of age and symbolic experience, children only map nonword symbols to referents when they are presented in typical social-referential contexts.
For this reason, we chose to embed the sound labels within common linguistic frames. The frames used in Experiment 1 differed slightly from those used in previous research (e.g., Woodward \& Hoyne, 1999); however, Namy and Waxman (2000) demonstrated that children map nonword symbols to referents when they are presented in the linguistic frames used in this study in addition to those used in previous research.

Next, the experimenter placed in the middle of the table a familiar object, one that may or may not have been used in the pretest. Children were allowed to play briefly with the object. The experimenter then removed the familiar object from the table. This was done to prevent children from perseverating on the novel objects, or their labels, between presentations.

The experimenter then placed a second novel object in the middle of the table. Again, children were allowed to play briefly with the object. The experimenter pointed to the object and directed children's attention to it, using speech, by saying, "Look at this one!" or "Oh! This one." The novel object was never labeled. This was repeated five times in total; the object was then removed from the table.

Next, the experimenter placed another familiar object on the table, one different from the familiar object used earlier in the trial. Children were allowed to play briefly with the object; the experimenter then removed it from the table.

The training procedure was repeated for a second time using the same labeled and unlabeled novel objects, as well as the same familiar objects. Thus, during a training trial children were exposed to the labeled novel object twice and heard its sound label repeated 10 times. They were exposed to the unlabeled object twice and were directed to look at it 10 times.

Testing followed immediately after training. The experimenter began testing by placing three objects in the middle of the table: (a) the labeled novel object, (b) the unlabeled novel object, and (c) a distractor novel object, a previously unseen novel object, also selected from the pool of 16 . The labeled and unlabeled novel objects were the same objects from the preceding training trial.

Children were allowed to play briefly with the objects. The experimenter asked children to select the labeled novel object from the array by saying, "Can you give me the [whoosh]?" or "Which one of these is the [whoosh]?" Only neutral feedback (e.g., "Thank you!") was provided to children when they made their selection. 
To complete the sound label task, the labelingtesting trail procedure was repeated three additional times with different novel objects, familiar objects, and sound labels.

\section{Results and Discussion}

\section{Comparison of Participant Characteristics}

A series of two-way analyses of variance (ANOVAs) were conducted to compare participants' age (in months) between the age and symbolic experience groups. Results indicated that there were statistically significant differences in age (in months) between younger and older age groups, $F(2$, $89)=126.97, \quad p<.05$. Regardless of symbolic experience, older children $(M=24.04$ months) were significantly older than younger children $(M=17.61$ months $)$. Results also indicated that there were statistically significant differences in age (in months) between the symbolic experience groups, $F(2,69)=3.72, p<.05$. Post hoc analyses indicated that monolingual children were significantly older ( $M=21.42$ months) than both unimodal bilingual children $(M=20.86$ months $)$ and children with bimodal symbolic experience $(M=20.45)$, least significant difference test (LSD), $p<.05$ for all comparisons. No other statistically significant effects were indicated.

To further investigate these differences, a series of one-way ANOVAs were conducted to compare age (in months) between the symbolic experience groups within each age group. Results indicated that for the younger children, there were statistically significant differences in age (in months) between the symbolic experience groups, $F(2,48)=6.96, p<.05$. Post hoc analyses indicated that younger monolingual children were significantly older $(M=18.14$ months) than younger unimodal bilingual children $(M=17.25$ months), as well as younger children with bimodal symbolic experience $(M=$ 17.06 months), LSD, $p<.05$ for all comparisons. No other statistically significant effects were indicated.

A second two-way ANOVA was conducted to compare participants' English vocabulary size between the age and symbolic experience groups. Results indicated that there were statistically significant differences in English vocabulary size between the age groups, $F(1,72)=67.63, p<.05$. Regardless of their symbolic experience, older children produced significantly more English words ( $M=107.59$ words) than did younger children $(M=384.09$ words). No other statistically significant effects were indicated.
A third two-way ANOVA was conducted to compare participants' sign vocabulary size between the age and symbolic experience groups. Results indicated that there were statistically significant differences in sign vocabulary size between the symbolic experience groups, $F(2,86)=18.59, p<.05$. Post hoc analyses indicated that regardless of their age group, children with bimodal symbolic experience produced significantly more signs $(M=24.54$ signs) than did monolingual children $(M=.56$ signs $)$ and unimodal bilingual children $(M=2.28$ signs), LSD, $p<.05$ for all comparisons. No other statistically significant effects were indicated.

Taken together, these results suggest that there were expected and desired similarities and differences in participant characteristics between the age and symbolic experience groups. Because vocabulary size serves as proxy measure of experience in word learning, and experience in word learning negatively correlates with symbolic flexibility (Acredolo \& Goodwyn, 1988; Huttenlocher, Haight, Bryk, Seltzer, \& Lyons, 1991; Namy et al., 2004), it was important that English vocabulary size be comparable between the symbolic experience groups. Results suggest that this was indeed the case: Although there were significant differences in English vocabulary size between younger and older children in general (which were to be expected based on the fact that age positively correlates with vocabulary size; Bloom, 1973), these differences were not present between younger and older children in the symbolic experience groups.

Furthermore, the fact that there were significant differences in age between younger and older children, and in sign vocabulary size between children with monolingual, unimodal bilingual, and bimodal symbolic experience, suggests that our placement of children in symbolic experience groups based on parental response was valid. Indeed, the only unexpected differences in participant characteristics were those found in age (in months) between the symbolic experience groups. We discuss the implications of these differences in detail in the General Discussion.

\section{Participant Characteristics and Performance on the Sound Label Task}

A series of Pearson product-moment correlations were conducted to compare age (in months), English vocabulary size, and sign vocabulary size to performance on the sound label task. Results indicated that there was a significant positive correlation between younger children's English vocabulary size and their 
performance on the sound label task, regardless of their symbolic experience, $r=.33, p<.05$. As younger children's English vocabulary size increased, so too did their performance on the sound label task. No other statistically significant correlations were indicated.

This finding was somewhat surprising given that previous research has demonstrated an inverse relation between monolingual children's English vocabulary size and their symbolic flexibility (e.g., Acredolo \& Goodwyn, 1988). This difference might be understood in terms of task demands. The sound label task appears to have been somewhat challenging for younger children. For example, mean scores for younger children in each symbolic experience group were low in comparison to the mean scores for older children in the dual-symbolic experience groups. Furthermore, anecdotally, younger children took slightly longer to complete the task than older children. Training and testing trials were always conducted in English; it is possible that knowing more English words allowed younger children to overcome task difficulties (e.g., hearing a digitized sound played from a hidden computer, understanding the directions of the experimenter) and thus promoted their performance on the sound label task.

Performance on the Sound Label Task According to Age and Symbolic Experience

Figure 2 depicts mean scores on the sound label task for younger $(M=2.06, S D=1.08)$ and older $(M=1.13, S D=.99)$ monolingual children, younger

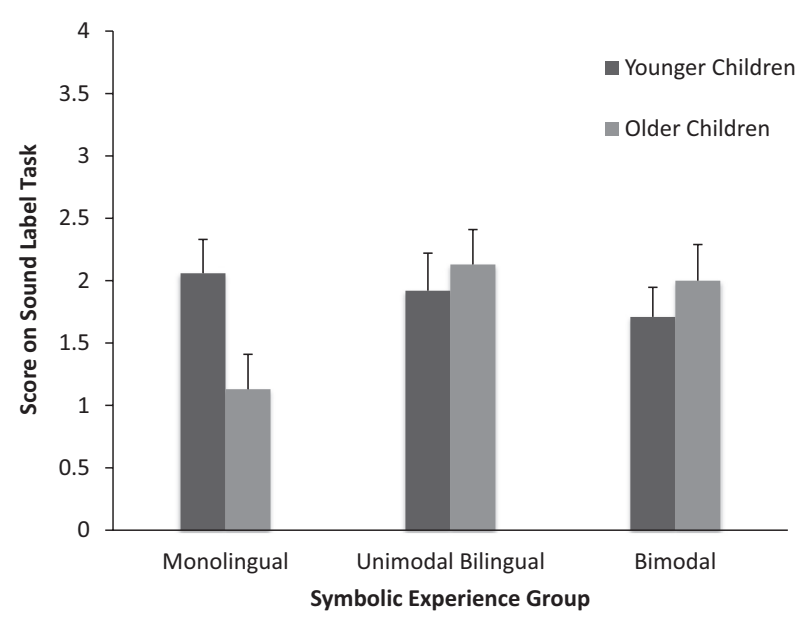

Figure 2. Mean score on the sound label task in Experiment 1 according to age and symbolic experience groups. Bars represent standard error.
$(M=1.92, S D=1.17)$ and older $(M=2.13, S D=$ 1.03) unimodal bilingual children, and younger $(M=1.71, \quad S D=.89)$ and older $(M=2.00$, $S D=1.03)$ children with bimodal symbolic experience. As can be seen, children's performance appears to have varied as a product of both their age and symbolic experience. A two-way ANOVA was conducted to test for this apparent interaction. Results confirmed that there was a statistically significant interactive effect of age and symbolic experience on performance on the sound label task, $F(2,88)=3.06, p=.05$. Post hoc analyses indicated that older unimodal children scored significantly higher on the sound label task than older monolingual children, LSD, $p<.05$. Older children with bimodal symbolic experience also scored significantly higher on the sound label task than older monolingual children, LSD, $p<.05$. No other statistically significant effects were indicated.

To explore further these differences, a series of planned independent sample $t$ tests were conducted to compare performance between the age groups within each symbolic experience group. Results indicated that younger monolingual children scored significantly higher on the sound label task than older monolingual children, $t(29)=2.60, p<.05$. No other statistically significant effects were indicated.

To examine the strength of children's performance on the sound label task, a series of onesample $t$ tests were conducted to compare performance of younger and older children in each symbolic experience group to chance. If children were responding randomly during testing, they would have been expected to score 1.32 (i.e., chance on each trial $=.33 \times 4$ trials $=1.32$ ). Results indicated that younger monolingual children performed at levels above what would be expected by chance, $t(15)=2.98, p<.05$. Younger unimodal bilingual children performed at levels marginally above what would be expected by chance, $t(13)=1.73, p=.10$, and older unimodal bilingual children performed at levels above what would be expected by chance, $t(14)=2.97, p<.05$. Older children with bimodal symbolic experience also performed at levels above what would be expected by chance, $t(13)=2.45$, $p<.05$. No other statistically significant effects were indicated.

Recall that for Experiment 1, we predicted that older children's symbolic flexibility would vary according to the breadth of their symbolic experience. Specifically, we predicted that older monolingual children would perform on the sound label task in ways indicative of a growing preference for words, whereas older children with dual-symbolic 
experience would perform in ways indicative of having intact symbolic flexibility. Results supported this hypothesis: Older unimodal bilingual children and children with bimodal symbolic experience outperformed older monolingual children. Furthermore, older unimodal bilingual children and older children with bimodal symbolic experience performed at above-chance levels, whereas older monolingual children did not. This pattern of results suggests that both unimodal bilingual and bimodal symbolic experience might maintain symbolic flexibility throughout early development, and presents two different interpretations regarding the mechanism underlying this phenomenon. We discuss both possibilities in detail in the General Discussion.

\section{Experiment 2}

Method

\section{Participants}

Participants were recruited for Experiment 2 in the same manner they had been for Experiment 1 . Assessment tools were identical between Experiments 1 and 2, as were the inclusion criteria for each symbolic experience group. The noteworthy difference between the samples was the number of age groups included. Because only older children exhibited differences in performance on the sound label task in Experiment 1, only older children were included in Experiment 2.

Table 1 presents a thorough summary of participant characteristics for the symbolic experience groups in Experiment 2. As can be seen, 47 children in total participated in Experiment 2: Fourteen children were included in the monolingual symbolic experience group $(M=23.27$ months of age, $M=95.08 \%$ English exposure), 19 children were included in the unimodal bilingual symbolic experience group $(M=23.12$ months of age, $M=50.46 \%$ English exposure, $M=49.23 \%$ Spanish exposure), and 14 children were included in the bimodal symbolic experience group $(M=$ 23.22 months of age, $M=93.10 \%$ English exposure). The sources of unimodal bilingual and bimodal symbolic experience were similar to those in Experiment 1.

\section{Materials and Procedure}

The pools of familiar and novel objects used in Experiment 2 were the same as those used in Experiment 1.
Recall that the key difference between Experiments 1 and 2 was the symbolic form used to label the novel objects in the training-testing trials of the symbol learning tasks. In Experiment 2, novel, English-like words were used to label the novel objects. The pool of words included: dax, gazzer, fez, fendle, pimwit, speff, brillig, tupa, jub, bluck, zav, and snarp.

Like the sound label task in Experiment 1, the word label task in Experiment 2 consisted of four training-testing trials. During training, the experimenter said, "This is the dax!" or "Look at the dax!" During testing, the experimenter said, "Can you give me the dax?" or "Which one of these is the dax?" In all other aspects, the methodology for the sound label and word label tasks was identical. The methodology for the pretests was also identical.

\section{Results and Discussion}

\section{Comparison of Participant Characteristics}

A series of one-way ANOVAs were conducted to compare participants' age (in months), English vocabulary size, and sign vocabulary size between the symbolic experience groups. Results indicated that there were statistically significant differences in children's sign vocabulary size between the symbolic experience groups, $F(2,44)=13.71, p<.05$. Post hoc analyses indicated that children with bimodal symbolic experience produced significantly more signs ( $M=11.27$ signs) than both monolingual children $(M=.69$ signs $)$ and unimodal bilingual children ( $M=1.29$ signs), LSD, $p<.05$ for both comparisons. This difference was expected based on our inclusion criteria for the symbolic experience groups. Recall that a similar effect was found in Experiment 1. No other effects were indicated.

\section{Participant Characteristics and Performance}

A series of Pearson product-moment correlations were conducted to compare participants' age (in months), English vocabulary size, and sign vocabulary size to performance on the word label task. Results indicated that there was a significant positive correlation between age and performance on the word label task, regardless of children's symbolic experience, $r=.28, p<.05$. As children's age (in months) increased, so too did their performance on the word label task. This finding was consistent with research indicating that age positively correlates with word learning ability (e.g., Bloom, 1973; Heibeck \& Markman, 1987). 
Performance on the Word Label Task Between Symbolic Experience Groups

A one-way ANOVA was conducted to compare performance on the word label task between the symbolic experience groups. Results indicated that there were no statistically significant differences in performance on the word label task between older monolingual children, older unimodal bilingual children, and older children with bimodal bilingual experience.

To examine the strength of children's performance on the word label task, a series of onesample $t$ tests were conducted to compare performance of children in each symbolic experience group to chance. If children were responding randomly during testing, they would have been expected to receive a score of $1.32 / 4$ (i.e., chance on each trial $=.33 \times 4$ trials $=1.32$ ). Results indicated that children in each symbolic experience group performed on the word label task at levels above what would be expected by chance; for monolingual children $(M=2.71, S D=1.33), t(13)=3.93$, $p<.05$; for unimodal bilingual children $(M=2.68$, $S D=1.10), t(18)=5.37, p<.05$; and for children with bimodal symbolic experience $(M=3.00$, $S D=1.11), t(13)=5.67, p<.05$.

Recall that for Experiment 2, we predicted that older children's general symbolic ability, as measured by performance on the word label task, would not vary according to the breadth of their symbolic experience. Results supported this hypothesis: Older monolingual children, older unimodal bilingual children, and older children with bimodal symbolic experience performed equally well on the word label task, at levels above what would be expected by chance. These results suggest that dual-symbolic experience had no impact on children's willingness to treat symbols from any form as labels for novel objects. In doing so, they help to rule out the possibility that the differences in performance on the sound label task in Experiment 1 resulted from differences in general symbolic ability, and lend further support to the idea that dual-symbolic experience might maintain children's symbolic flexibility throughout early development.

\section{General Discussion}

In this study, we examined how differences in children's symbolic experience might lead to differences in the developmental trajectory of their symbolic flexibility. We did so in an effort, first, to explore the relation between symbolic experience and symbolic flexibility throughout early development, and second, to understand better the factors contributing to monolingual children's temporary preference for words.

Consistent with previous research (e.g., Namy \& Waxman, 1998; Petitto et al., 2012; Woodward \& Hoyne, 1999), we observed a decrease in the symbolic flexibility of children with monolingual symbolic experience between the ages of 18 and 24 months. In contrast, we observed relative consistency in the symbolic flexibility of children with dual-symbolic experience over the same period of time. At 24 months of age, children with dual-symbolic experience exhibited greater symbolic flexibility than children with monolingual symbolic experience. This finding is consistent with a large body of research demonstrating that bilingual children with outperform monolingual children on a wide variety of cognitive and language learning tasks, as well as tests of representational flexibility (Adi-Japha et al., 2010; Bialystok \& Martin, 2004; Bialystok \& Shapero, 2005; Bialystok \& Viswanathan, 2009; Brito \& Barr, 2012; Kovács \& Mehler, 2009a, 2009b).

\section{Symbolic Experience Shapes Symbolic Flexibility}

Whereas previous research has suggested that children's symbolic flexibility depends on their developmental level, the results of our study suggest that it also depends on the breadth of their symbolic experience. Our results lend preliminary support to the idea that the development of monolingual children's temporary preference for words is driven by their (temporarily) narrow symbolic experience. Their representational systems, although initially nondiscriminatory, might attune over time to the symbolic form that has been predominant in their symbolic experience, and might also learn to expect that symbolic form in new symbol learning contexts (Eimas et al., 1971; Palmer et al., 2012; Tees \& Werker, 1984). However, the process of adaptation might be more continuous than finite: Monolingual children's representational systems might readapt as their symbolic experience naturally accumulates and diversifies, and their need for symbolic flexibility eclipses their need for a preference for words.

Interestingly, our results suggest that neither children with unimodal bilingual symbolic experience nor children with bimodal symbolic experience develop a preference for words in the same manner as monolingual children. The question remains how 
dual-symbolic experience might maintain children's symbolic flexibility throughout early development. Recall that there were two possible interpretations for our pattern of results. The first was that older children's symbolic flexibility had results from abilities uniquely associated with each type of dual-symbolic experience. In other words, different abilities had led to the same liberal mapping behavior. For example, greater efficiency in environmental monitoring, and in attentional control (Bialystok \& Shapero, 2005; Comeau \& Genesee, 2001; Costa et al., 2008), might have made older children with unimodal bilingual experience more likely to utilize the social and contextual cues to reference in the sound label task (e.g., the use of common linguistic frame, the eye gaze of the experimenter, the pointing to or movement of the objects; Yow \& Markman, 2011), and thus more likely to map the sound labels to the novel objects. In contrast, a heightened awareness of the function of all symbolic forms, or increased tendency to make conceptual abstractions (Namy \& Gentner, 2002; Oakes \& Ribar, 2005; Waxman, 2003; Waxman \& Klibanoff, 2000), might have made older children with bimodal symbolic experience more likely to generalize their previous symbol learning experience to the new symbol learning contexts of the sound label task (Brojde \& Colunga, 2006; Colunga \& Smith, 2002) and thus more likely to map the sound labels to the novel objects.

The second interpretation of our pattern of results was that older children's symbolic flexibility had resulted from abilities associated with both types of dual-symbolic experience. For example, older children with unimodal bilingual experience and older children with bimodal symbolic experience might have similarly benefited from a heightened awareness of the function of all symbols and an increased tendency to make conceptual abstractions and higher level generalizations. Previously, we had argued that these abilities might be uniquely associated with bimodal bilingualism. However, it could be the case that they result from regular exposure to multiple symbolic forms, regardless of the timing of their presentation and use.

The design of our study prevents us from precisely identifying the mechanism(s) underlying symbolic flexibility of older children with dual-symbolic experience. Given the differences in the experience and abilities of unimodal and bimodal bilingual children (Emmorey, Borinstein, et al., 2008; Emmorey, Luk, et al., 2008; Pyers et al., 2009), the former explanation, although less parsimonious, is perhaps more accurate. The possibility remains, however, that there are more commonalities in the experience and abilities of children with unimodal bilingual symbolic experience and children with bimodal symbolic experience, and by extension, of unimodal bilingual children and true bimodal bilingual children, than previously thought. In this context, the inclusion of children with bimodal symbolic experience, rather than true bimodal bilingual children, proves to be more illuminating than limiting: The performance of this group suggests that intact symbolic flexibility in later early development does not depend on exposure to the grammatical elements of multiple languages. What appears to be key is exposure to and use of multiple symbolic forms, and this is consistent with the explanations we have outlined here.

In proposing these explanations, we have primarily focused on how abilities associated with dualsymbolic experience might help older children to override a preference for the symbolic forms to which they have been regularly exposed. However, it could be the case that the representational systems of children with dual-symbolic experience develop in fundamentally different-and less understoodways than those of children with monolingual symbolic experience. For example, dual-symbolic experience might completely interrupt the process of adaptation and attenuation of children's representational systems (Petitto et al., 2012). For all these reasons, our results create important avenues for future research: What is the mechanism underlying symbolic flexibility in older children with dual-symbolic experience? What similarities, if any, exist between the experience and abilities of children with different types of dual-symbolic experience or children with different types of bilingual symbolic experience? How might dual-symbolic experience and bilingual symbolic experience uniquely engage, and shape, children's representational systems? These questions have yet to be answered.

\section{Considerations}

We cannot conclude with certainty that differences in the breadth of children's symbolic experience lead to differences in the development trajectory of their symbolic flexibility due to the quasi-experimental design of our study. Other factors might have systematically varied between the age and symbolic experience groups, and thus alternately account for our pattern of results. One such factor might have been the age of children in the symbolic experience groups: Recall that monolingual children in Experiment 1 were significantly older than children with dual-symbolic experience, despite 
our best efforts to match relevant participant characteristics between the symbolic experience groups.

Because age is typically inversely related to symbolic flexibility for monolingual children (Namy \& Waxman, 1998; Namy et al., 2004; Woodward \& Hoyne, 1999), it is possible that the older monolingual children in Experiment 1 were less likely to map sounds to novel objects in the sound label task than older children with dual-symbolic experience as the result of their more advanced age, not their more narrow symbolic experience. However, there are a number of reasons why this possibility is unlikely. First, age was not related to performance on the sound label task. Second, follow-up analyses suggested that differences in age primarily existed between younger children in the symbolic experience groups (who performed equally well on the sound label task). Finally, the mean ages of the older children in each symbolic experience groups were well within the period of development when a preference for words, if present, should be exhibited (Namy \& Waxman, 1998; Namy et al., 2004; Woodward \& Hoyne, 1999). If we had examined the symbolic flexibility of each symbolic experience group independently, it would have been significant if older children in any of the groups performed at levels above what would be expected by chance on the sound label task.

In addition, unimodal bilingual children in Experiments 1 and 2 appeared to have had larger English vocabulary sizes than Spanish vocabulary sizes (note that a direct statistical comparison was not possible because the English and Spanish versions of the MCDI are different measures). Because vocabulary size is considered to be a proxy measure of amount of exposure to a language (Huttenlocher et al., 1991), a difference in English and Spanish vocabulary sizes, if present, might suggest that the children in our experiments were not true unimodal bilinguals. This, in turn, might suggest that a factor other than symbolic experience contributed to the observed differences in performance on the sound label task. Again, this possibility is unlikely. For unimodal bilingual children, vocabulary size is a less reliable measure of exposure to and knowledge of a language (Gollan, Montoya, Cera, \& Sandoval, 2008). Furthermore, similar studies (e.g., Brito \& Barr, 2012) have demonstrated that amount of exposure to each language is a better predictor of cognitive flexibility in bilingual populations than is vocabulary size in each language. For this reason, we were careful to adhere strictly to our inclusion criteria when placing children into symbolic experience groups, to ensure that exposure to each language was comparable between unimodal bilingual children. Our statistical comparisons of participant characteristics suggest that this was accomplished.

\section{Significance of Work}

Although other studies (e.g., Namy \& Waxman, 1998) have suggested that a relation exists between symbolic experience and symbolic flexibility, ours is the first to do so by comparing different types of symbolic experience as they occur in the real world rather than by manipulating symbolic experience in tightly controlled laboratory settings. Our study is also the first to suggest that experience in learning multiple symbolic forms generalizes to contexts in which children are asked to learn symbols from untrained forms as labels for referents. In putting forth these suggestions, our study provides important preliminary support for two suppositions: (a) the idea that the breadth of symbolic experience dictates the developmental trajectory of symbolic flexibility and (b) a new account of the variability in monolingual children's symbolic flexibility in early development, although future research is needed to test its assumptions with monolingual children.

Our study also adds to the large body of research that highlights the adaptability of the young learner and their various cognitive systems (e.g., Eimas et al., 1971; Palmer et al., 2012; Smith et al., 2002). Our results suggest that as a result of the adaptability of their representational systems, many different developmental trajectories of symbolic flexibility are possible-including those that were demonstrated in this study, as well as others that have yet to be examined. This is significant, as it encourages us to consider a larger swath of the population when charting the course of symbolic development. In the United States, it is becoming increasingly common for children to be exposed to multiple symbolic forms during the first few years of life (U.S. Census Bureau, 2009). And, on a global level, monolingual children are in the minority: Two thirds of children around the world are raised in bilingual homes (Crystal, 1997). What we have considered to be the "typical" course of symbolic development might be just the opposite.

\section{References}

Acredolo, L., \& Goodwyn, S. (1988). Symbolic gesturing in normal infants. Child Development, 59, 450-466. doi: $10.2307 / 1130324$ 
Adi-Japha, E., Berberich-Artzi, J., \& Libnawi, A. (2010). Cognitive flexibility in drawings of bilingual children. Child Development, 81, 1356-1366. doi:10.1111/j. 1467-8624.2010.01477.x

Baldwin, D. A. (1993). Infants' ability to consult the speaker for clues to word reference. Journal of Child Language, 20, 395-418. doi:10.1017/S0305000900008345

Behrens, H. (2006). The input-output relationship in first language acquisition. Language and Cognitive Processes, 21, 2-24. doi:10.1080/01690960400001721

Bialystok, E., Craik, F. M., \& Ryan, J. (2006). Executive control in a modified anti-saccade task: Effects of aging and bilingualism. Journal of Experimental Psychology: Learning, Memory, and Cognition, 32, 1341-1354. doi: 10.1037/0278-7393.32.6.1341

Bialystok, E., \& Martin, M. M. (2004). Attention and inhibition in bilingual children: Evidence from the dimensional change card sort task. Developmental Science, 7, 325-339. doi:10.1111/j.1467-7687.2004.00351.x

Bialystok, E., \& Shapero, D. (2005). Ambiguous benefits: The effect of bilingualism on reversing ambiguous figures. Developmental Science, 8, 595-604. doi:10.1111/j. 1467-7687.2005.00451.x

Bloom, L. (1973). One word at a time: The use of singleword utterances before syntax. Hague, Netherlands: Mouton.

Brito, N., \& Barr, R. (2012). Influence of bilingualism on memory generalization during infancy. Developmental Science, 15, 812-816. doi:10.1111/j.1467-7687.2012.1184.x

Brojde, C., \& Colunga, E. (2006). Out of the mouths of babes. Proceedings of the Annual Conference of the Cognitive Science Society, 27, 223-229.

Campbell, A. L., \& Namy, L. L. (2003). The role of socialreferential context in verbal and nonverbal symbol learning. Child Development, 74, 549-563. doi:10.1111/ 1467-8624.7402015

Colunga, E., \& Smith, L. B. (2002). What makes a word? Proceedings of the Annual Conference of the Cognitive Science Society, 24, 214-219.

Comeau, L., \& Genesee, F. (2001). Bilingual children's repair strategies during dyadic communication. In J. Cenoz \& F. Genesee (Eds.), Trends in bilingual acquisition (pp. 229-256). Amsterdam, Netherlands: John Benjamins.

Costa, A., Hernández, M., \& Sebastián-Gallés, N. (2008). Bilingualism aids conflict resolution: Evidence from the ANT task. Cognition, 106, 59-86. doi:10.1016/j.cognition.2006.12.013

Crystal, D. (1997). English as a global language. Cambridge, UK: Cambridge University Press.

Eimas, P. D., Sigueland, E. R., Jusczyk, P., \& Vigorito, J. (1971). Speech perception in infants. Science, 17, 303306. doi:10.1126/science.171.3968.303

Emmorey, K., Borinstein, H. B., Thompson, R., \& Gollan, T. H. (2008). Bimodal bilingualism. Bilingualism: Language and Cognition, 11, 43-61. doi:10.1017/S1366728907003203

Emmorey, K., Luk, G., Pyers, L., \& Bialystok, E. (2008). The source of enhanced cognitive control in bilinguals:
Evidence from bimodal bilinguals. Psychological Science, 19, 1201-1206. doi:10.1111/j.1467-9280.2008.02224.x

Fenson, L., Dale, P. S., Resnick, J. S., Thal, D., Bates, E., Hartung, J. P., et al. (1993). The MacArthur-Bates Communicative Development Inventories. San Diego, CA: Singular.

Gollan, T. H., Montoya, R. I., Cera, C., \& Sandoval, T. C. (2008). More use almost always means a smaller frequency effect: Aging, bilingualism, and the weaker links hypothesis. Journal of Memory and Language, 58, 787-814. doi:10.1016/j.jml.2007.07.001

Heibeck, T. H., \& Markman, E. M. (1987). Word learning in children: An examination of fast mapping. Child Development, 58, 1021-1034.

Hollich, G. J., Hirsh-Pasek, K., Golinkoff, R., Brand, R. J., Brown, E., Chung, H., et al. (2000). Breaking the language barrier: An emergentist coalition model for the origins of word learning. Monographs of the Society for Research in Child Development, 65(3, Serial No. 262).

Huttenlocher, J., Haight, W., Bryk, A., Seltzer, M., \& Lyons, T. (1991). Early vocabulary growth: Relation to language input and gender. Developmental Psychology, 27, 236-248. doi:10.1037/0012-1649.27.2.236

Iverson, J. M., Capirci, O., Longobardi, E., \& Caselli, M. (1999). Gesturing in mother-child interactions. Cognitive Development, 14, 57-75. doi:10.1016/S0885-2014(99) 80018-5

Kovács, Á., \& Mehler, J. (2009a). Flexible learning of multiple speech structures in bilingual infants. Science, 325, 611-612. doi:10.1126/science.1173947

Kovács, Á., \& Mehler, J. (2009b). Cognitive gains in 7-month-old bilingual infants. Proceedings of the National Academy of Sciences of the United States of America, 106, 6556-6560. doi:10.1073/pnas.0811323106

Kovelman, I., Shalinsky, M. H., White, K. S., Schmitt, S. N., Berens, M. S., Paymer, N., et al. (2009). Dual language use in sign-speech bimodal bilinguals: fNIRS brain-imaging evidence. Brain and Language, 109, 112-123. doi:10.1016/j.band1.2008.09.008

Mervis, C. B., \& Bertrand, J. (1995). Early lexical acquisition and the vocabulary spurt: A response to Goldfield \& Reznick. Journal of Child Language, 22, 461-468. doi:10.1017/S0305000900009880

Namy, L. L. (2001). What's in a name when it isn't a word? 17-month-olds' mapping of nonverbal symbols to object categories. Infancy, 2, 73-86. doi:10.1207/ S15327078IN0201_5

Namy, L. L., Acredolo, L., \& Goodwyn, S. (2000). Verbal labels and gestural routines in parental communication with young children. Journal of Nonverbal Behavior, 24, 63-79. doi:10.1023/A:1006601812056

Namy, L. L., Campbell, A. L., \& Tomasello, M. (2004). The changing role of iconicity in non-verbal symbol learning: A U-shaped trajectory in the acquisition of arbitrary gestures. Journal of Cognition and Development, 5, 37-57. doi:10.1207/s15327647jcd0501_3

Namy, L. L., \& Gentner, D. (2002). Making a silk purse out of two sow's ears: Young children's use of compari- 
son in category learning. Journal of Experimental Psychology: General, 131, 5-15.

Namy, L. L., \& Waxman, S. R. (1998). Words and gestures: Infants' interpretation of different forms of symbolic reference. Child Development, 69, 295-308. doi: $10.2307 / 1132165$

Namy, L. L., \& Waxman, S. R. (2000). Naming and exclaiming: Infants' sensitivity to naming contexts. Journal of Cognition and Development, 1, 405-428. doi:10. 1207/S15327647JCD0104_03

Nelson, K. (1973). Structure and strategy in learning to talk. Monographs of the Society for Research in Child Development, 38(1-2, Serial No. 149). doi:10.2307/ 1165788

Oakes, L. M., \& Ribar, R. J. (2005). A comparison of infants' categorization in paired and successive presentation familiarization tasks. Infancy, 7, 85-98.

Palmer, S. B., Fais, L., Golinkoff, R. M., \& Werker, J. F. (2012). Perceptual narrowing of linguistic sign occurs in the 1st year of life. Child Development, 83, 543-553. doi:10.1111/j.1467-8624.2011.01715.x

Petitto, L. A., Berens, M. S., Kovelman, I., Dubins, M. H., Jasinska, K., \& Shalinksy, M. (2012). The "perceptual wedge hypothesis" as the basis for bilingual babies phonetic processing advantage: New insights from fNIRS brain imaging. Brain and Language, 121, 142-155. doi:10.1016/j.bandl.2011.05.003

Piaget, J. (1997). The language and thought of the child. In H. E. Gruber \& J. J. Vonèche (Eds.), The essential Piaget: An interpretive reference and guide (pp. 65-88). New York: Basic Books. (Original work published 1923).

Pyers, J. E., Gollan, T. H., \& Emmorey, K. (2009). Bimodal bilinguals reveal the source of tip- of-the-tongue states. Cognition, 112, 323-329. doi:10.1016/j.cognition.2009.04. 007
Samuelson, L. K., \& Smith, L. B. (2005). They call it like they see it: Spontaneous naming and attention to shape. Developmental Science, 8, 182-198.

Scaife, M. M., \& Bruner, J. S. (1975). The capacity for joint visual attention in the infant. Nature, 253, 265-266. doi: $10.1038 / 253265 \mathrm{a} 0$

Smith, L. B., Jones, S. S., Landau, B., Gershkoff-Stowe, L., \& Samuelson, L. (2002). Object name learning provides on-the-job training for attention. Psychological Science, 13, 13-19. doi:10.1111/1467-9280.00403

Tees, R. C., \& Werker, J. F. (1984). Perceptual flexibility: Maintenance or recovery of the ability to discriminate non-native speech sounds. Canadian Journal of Psychology/Revue Canadienne De Psychologie, 38, 579-590. doi: $10.1037 /$ h0080868

Tomasello, M. (1992). The social bases of language acquisition. Social Development, 1, 67-87. doi:10.1111/j. 1467-9507.1992.tb00135.x

U.S. Census Bureau. (2009). American Community Survey. Washington, DC: Author.

Waxman, S. R. (2003). Links between object categorization and naming: Origins and emergence in human infants. In D. H. Rakison \& L. M. Oakes (Eds.), Early category and concept development: Making sense of the blooming, buzzing confusion (pp. 213-241). New York: Oxford University Press.

Waxman, S. R., \& Klibanoff, R. S. (2000). The role of comparison in the extension of novel adjectives. Developmental Psychology, 36, 571-581.

Woodward, A., \& Hoyne, K. L. (1999). Infants' learning about words and sounds in relation to objects. Child Development, 70, 65-77. doi:10.1111/1467-8624.00006

Yow, W., \& Markman, E. M. (2011). Young bilingual children's heightened sensitivity to referential cues. Journal of Cognition and Development, 12, 12-31. doi:10.1080/ 15248372.2011.539524 